\title{
Optical surgical navigation system causes pulse oximeter malfunction
}

\author{
Masaaki Satoh ${ }^{1}$, Tetsuhito Hara', Kenji Tamai ${ }^{1}$, Juntaro Shiba ${ }^{1}$, Kunihisa Hotta ${ }^{1}$, Mamoru Takeuchi ${ }^{1}$ \\ and Eiju Watanabe ${ }^{2}$
}

\begin{abstract}
An optical surgical navigation system is used as a navigator to facilitate surgical approaches, and pulse oximeters provide valuable information for anesthetic management. However, saw-tooth waves on the monitor of a pulse oximeter and the inability of the pulse oximeter to accurately record the saturation of a percutaneous artery were observed when a surgeon started an optical navigation system. The current case is thought to be the first report of this navigation system interfering with pulse oximetry. The causes of pulse jamming and how to manage an optical navigation system are discussed.
\end{abstract}

Keywords: Neuronavigator; Saw-tooth waves; Anesthetic management; Tracking technology; Laser

\section{Background}

The Polaris spectra optical measurement system (NDI, Waterloo, Canada) is a surgical navigation system used for CT-guided stereotaxic neurosurgery. The concept of neuronavigator was introduced in 1987 [1], and optical tracking systems have been in use since 2006 [2]. A neuronavigator facilitates neurosurgical approaches.

While a pulse oximeter is an essential monitor for anesthesia management, the malfunction of a pulse oximeter was observed several times during general anesthesia management of neurosurgery, and it was finally determined that it resulted from the navigation system, and a way to manage the problem was developed.

\section{Case presentation}

A 77-year-old man with a left brain tumor was scheduled for craniotomy. He had no relevant past history. The standard monitors were used. Anesthesia was gently induced and smoothly maintained with total intravenous anesthesia. Before the operation, the surgeon set up the Polaris spectra optical measurement system, and the pulse oximeter (NIHON KOHDEN, Tokyo, Japan) showed different values for oxygen saturation $\left(\mathrm{SpO}_{2}\right)$ and pulse rate on its display. When the pulse oximeter was affected, for

\footnotetext{
* Correspondence: anemasa@jichi.ac.jp

${ }^{1}$ Department of Anesthesiology and Critical Care Medicine, Jichi Medical University, Yakushiji, Shimotsuke-City, Tochigi 329-0498, Japan Full list of author information is available at the end of the article
}

example, the value of $\mathrm{SpO}_{2}$ changed from 99 to $94 \%$ and then from 94 to $100 \%$. This phenomenon was similarly confirmed by other pulse oximeters made by a different manufacturer (MASIMO Corp, Irvine, CA). Figure 1a shows that saw-tooth waves were produced with the waves of the pulse oximeter.

The cause of this anomaly was detected by careful observation of the surgeons' behavior. It was found that the Polaris spectra optical measurement system affected the plethysmogram. The abnormal waves varied when measured in different directions (Fig. 1b, c). Moreover, when a person passed between the Polaris spectra optical measurement system and the pulse oximeter, the phenomenon did not occur (Fig. 1d). The surgeon was then told that the Polaris spectra optical measurement system caused the pulse oximeter to malfunction, and the Polaris spectra optical measurement system was then arranged so that the surgeon was between the Polaris spectra optical measurement system and the pulse oximeter. Anesthesia was then uneventfully maintained, and the operation was successfully finished.

This appears to be the first report to indicate two crucial clinical findings related to optical surgical navigation systems: first, saw-tooth waves occur constantly during power-on, and second, that the interference can be eliminated by appropriate positioning during the operation.

The Polaris spectra optical measurement system (Fig. 2a, b) displays positional information on the monitor 


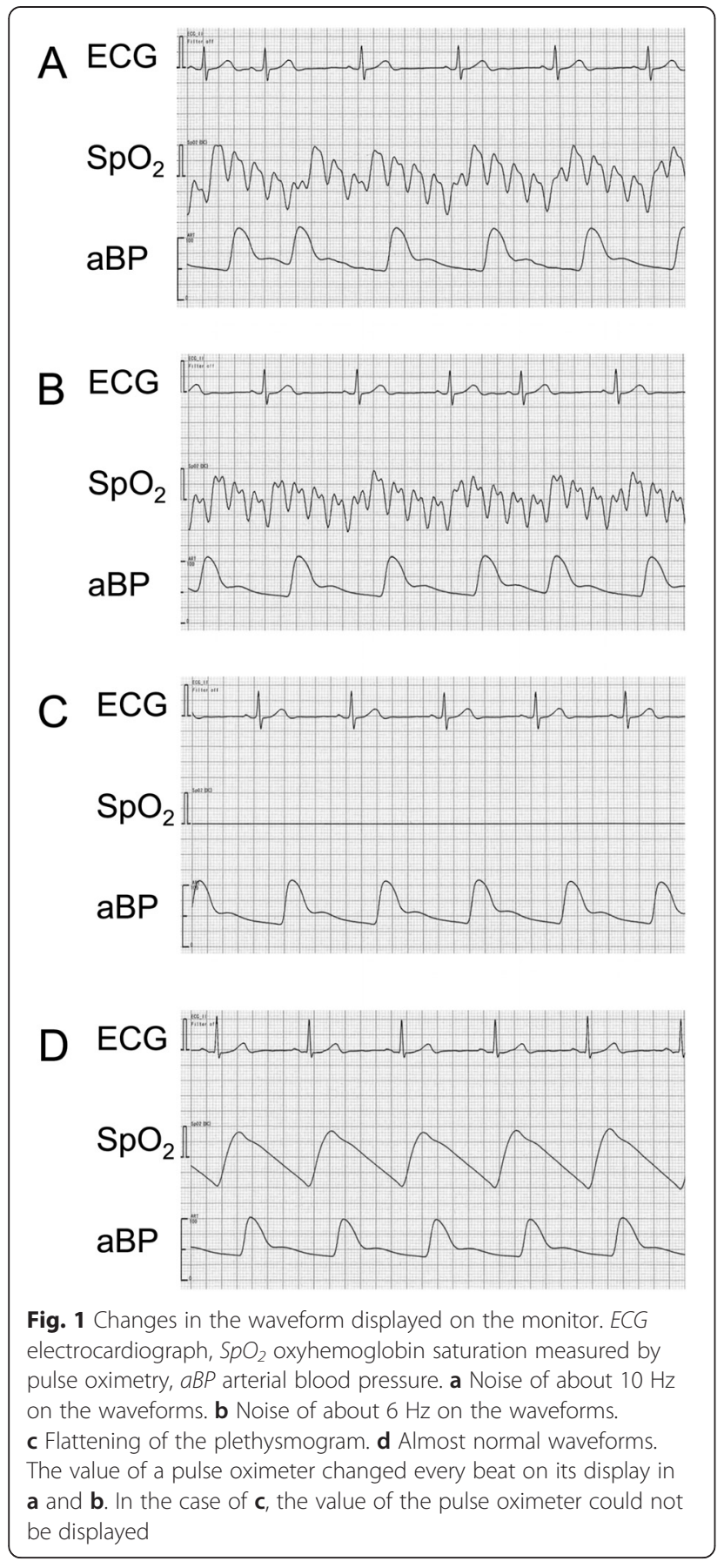

using three-dimensional measurement tracking technology via a positioning laser (class 2 laser, 635-nm wavelength). Moreover, the update rate in this system is $60 \mathrm{~Hz}$.

Currently available oximeters use two light-emitting diodes that emit light at wavelengths of $660 \mathrm{~nm}$ (red) and $940 \mathrm{~nm}$ (infrared). $\mathrm{O}_{2} \mathrm{Hb}$ and $\mathrm{Hb}$ have different absorption spectra at these particular wavelengths. In the red region, $\mathrm{O}_{2} \mathrm{Hb}$ absorbs less light than $\mathrm{Hb}$, while the reverse occurs in the infrared region [3]. Sources of error in the pulse oximeter are mainly known to involve four factors: inadequate
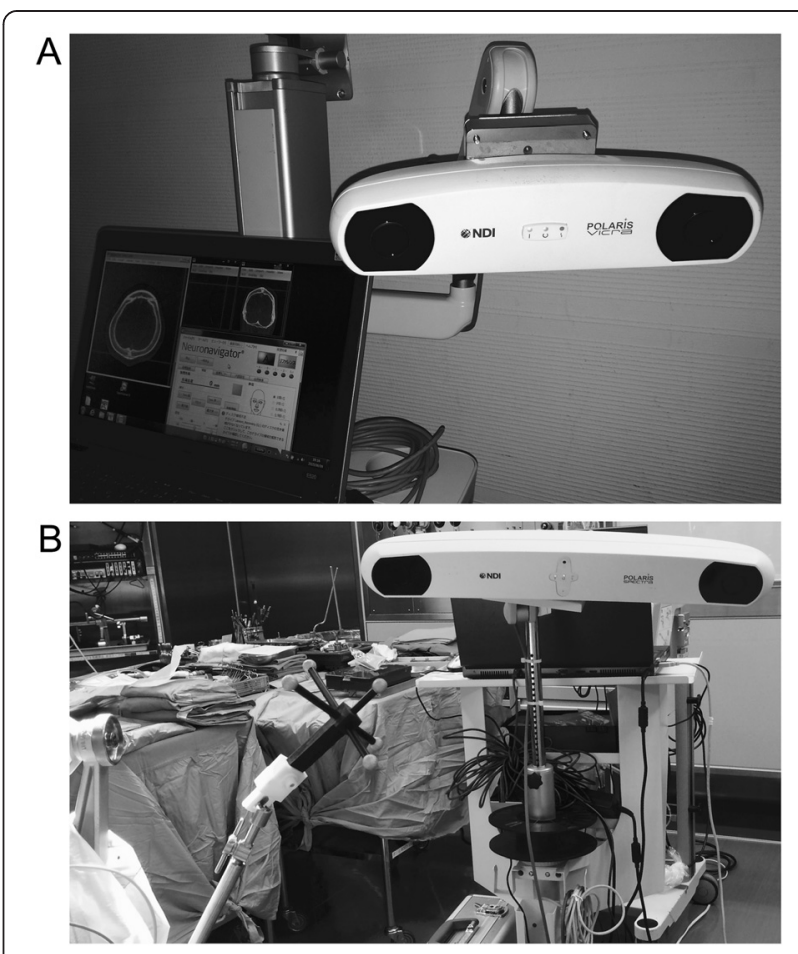

Fig. 2 The Polaris spectra optical measurement system. a The main unit: a Position sensor and a PC. $\mathbf{b}$ A reference frame and the main unit

attachment, noise affecting the absorbance curve, interference with the stability of an absorbance curve, and the existence of a material attenuating the transmitted light strength.

It was thought that the Polaris spectra optical measurement system caused noise affecting the absorbance curve of the pulse oximeter. The update rate of this tracking system may directly cause flattening of the waves and a $6-$ to $10-\mathrm{Hz}$ noise causing pulse jamming of the waveforms on the display of the pulse oximeter. In other words, the tracking system may have strongly affected the detection of the artery signal.

Moreover, the wavelength of the Polaris spectra optical measurement system is $635 \mathrm{~nm}$, which is close to the $660 \mathrm{~nm}$ of the red region. The Polaris spectra optical measurement system could thus enhance the absorbance of $\mathrm{Hb}$ rather than $\mathrm{O}_{2} \mathrm{Hb}$ in the red region. This effect can be eliminated almost entirely by placing a person between the Polaris spectra optical measurement system and the pulse oximeter. The authors present an alternative technique. This phenomenon disappears by wrapping the sensor of the pulse oximeter in aluminum foil. When the authors explained this phenomenon to a co-author, who first used the term neuronavigator [1], he suggested aluminum foil wrapping of the pulse oximeter. He said that he had noticed this at the time of animal experiments. It is significant for anesthesiologists to be aware of this phenomenon when using the Polaris spectra optical measurement system during surgery. 


\section{Conclusions}

The Polaris spectra optical measurement system, a neuronavigator, causes pulse oximeters to malfunction by pulse jamming. This phenomenon and its effects can be normalized by positioning a person between the Polaris spectra optical measurement system and the pulse oximeter or by wrapping the sensor of the pulse oximeter in aluminum foil.

\section{Consent}

Written informed consent was obtained from the patient for publication of this case report and any accompanying images. A copy of the written consent is available for review by the Editor-in-Chief of this journal.

\section{Competing interests}

The authors declare that they have no competing interests.

\section{Authors' contributions}

MS participated in the anesthetic management of this current case, conceived of the educational importance of this paper, and drafted the manuscript. TH participated in the anesthetic management of this current case and acquired the data. KT participated in the anesthetic management of this current case and analyzed the data. JS participated in the anesthetic management of this current case and acquired the data. $\mathrm{KH}$ helped to draft the manuscript. MT was involved in drafting the manuscript. EW was involved in revising it critically for important intellectual content. All authors read and approved the final manuscript.

\section{Author details}

${ }^{1}$ Department of Anesthesiology and Critical Care Medicine, Jichi Medical University, Yakushiji, Shimotsuke-City, Tochigi 329-0498, Japan. ²Department of Neurosurgery, Jichi Medical University, Yakushiji, Shimotsuke-City, Tochigi 329-0498, Japan.

Received: 20 May 2015 Accepted: 14 July 2015

Published online: 27 August 2015

\section{References}

1. Watanabe E, Watanabe T, Manaka S, Mayanagi Y, Takakura K. Three-dimensional digitizer (neuronavigator): new equipment for computed tomography-guided stereotaxic surgery. Surg Neurol. 1987;27:543-7.

2. Willems PWA, Berkelbach van der Sprenkel JW, Tulleken CAF, Viergever MA, Taphoorn MJB. Neuronavigation and surgery of intracerebral tumours. J Neurol. 2006;253:1123-36.

3. Jubran A. Pulse oximetry. In: Tobin MJ, editor. Principles and practice of intensive care monitoring. New York: McGraw Hill, Inc; 1998. p. 261-87.

\section{Submit your manuscript to a SpringerOpen ${ }^{\circ}$ journal and benefit from:}

- Convenient online submission

Rigorous peer review

- Immediate publication on acceptance

- Open access: articles freely available online

- High visibility within the field

- Retaining the copyright to your article

Submit your next manuscript at $>$ springeropen.com 